\title{
Image analysis for measuring the size stratification in sand-gravel laboratory experiments
}

\author{
C. Orrú ${ }^{1}$, V. Chavarrías ${ }^{1,2}$, W. S. J. Uijttewaal ${ }^{1}$, and A. Blom ${ }^{1}$ \\ ${ }^{1}$ Environmental Fluid Mechanics Section, Civil Engineering and Geosciences, Delft University of Technology, \\ P.O. Box 5048, 2600 GA, Delft, the Netherlands \\ ${ }^{2}$ Escola Tècnica Superior d'Enginyers de Camins, Canals i Ports de Barcelona, Universitat Politècnica de \\ Catalunya, C/Jordi Girona, 31, 08034 Barcelona, Spain \\ Correspondence to: C. Orrú (c.orru@tudelft.nl)
}

Received: 15 October 2013 - Published in Earth Surf. Dynam. Discuss.: 19 November 2013

Revised: 6 March 2014 - Accepted: 17 March 2014 - Published: 9 April 2014

\begin{abstract}
Measurements of spatial and temporal changes in the grain-size distribution of the bed surface and substrate are crucial to improving the modelling of sediment transport and associated grain-size selective processes. We present three complementary techniques to determine such variations in the grain-size distribution of the bed surface in sand-gravel laboratory experiments, as well as the resulting size stratification: (1) particle colouring, (2) removal of sediment layers, and (3) image analysis. The resulting stratification measurement method was evaluated in two sets of experiments. In both sets three grain-size fractions within the range of coarse sand to fine gravel were painted in different colours. Sediment layers are removed using a wet vacuum cleaner. Subsequently areal images are taken of the surface of each layer. The areal fraction content, that is, the relative presence of each size fraction over the bed surface, is determined using a colour segmentation algorithm which provides the areal fraction content of a specific colour (i.e. grain size) covering the bed surface. Particle colouring is not only beneficial to this type of image analysis but also to the observation and understanding of grain-size selective processes. The size stratification based on areal fractions is measured with sufficient accuracy. Other advantages of the proposed size stratification measurement method are (a) rapid collection and processing of a large amount of data, (b) a very high spatial density of information on the grain-size distribution, (c) the lack of disturbances to the bed surface, (d) only minor disturbances to the substrate due to the removal of sediment layers, and (e) the possibility to return a sediment layer to its original elevation and continue the flume experiment. The areal fractions are converted into volumetric fractions using an existing conversion model.
\end{abstract}

\section{Introduction}

Spatial and temporal variations in the grain-size distribution of the river bed surface are the outcome of grain-size selective processes and/or abrasion (e.g. Parker, 1991a, b). Having insight in such variations is crucial to understanding a river system and predicting the autonomous morphodynamic behaviour of a river system, as well as the morphodynamic effects of (future) human interventions. Spatial variations in the grain-size distribution are usually indicated with the term sorting. An example of streamwise sorting is downstream fining, which is characterized by a decrease in mean grain size in streamwise direction. Downstream fining is usually attributed to abrasion and grain-size selective transport (e.g. Parker, 1991a,b). An example of sorting in the vertical direction is armouring. Two types of armouring can be distinguished: static and mobile armour layers (e.g. Parker and Klingeman, 1982; Jain, 1990). A static armour forms at low shear stresses when the finer grains are mobile while the coarser ones are not, which under limited sediment supply conditions results in a coarsening of the bed surface. This coarsening prevents the underlying finer sediment from being entrained by the flow. Instead, a mobile armour develops at higher shear stresses at which coarser grains are 
also supplied from upstream. A coarse surface layer is then needed to increase the transport capacity of the coarse sediment which enables the sediment supplied from upstream to be transported downstream. Dune sorting is another example of vertical sorting, and originates through (a) avalanching of grains down the bedform lee face which results in an upward fining pattern (e.g. Bagnold, 1941; Allen, 1965) and (b) selective or partial transport, which can result in the formation of a coarse layer underneath the migrating dunes consisting of the less mobile sediment (e.g. Zanke, 1976; Blom et al., 2003). Sorting processes at local scale are also seen in bars, where coarser sediment tends to be found on bar heads and fines on bar tails (e.g. Leopold and Wolman, 1957; Ashworth and Ferguson, 1986; Lunt and Bridge, 2004). Lateral sorting can be observed in meander bends where the shallower inner bends are finer than the deeper outer bends (e.g. Parker and Andrews, 1985; Julien and Anthony, 2002). Monitoring techniques that allow for rapid and non-destructive measurement of sorting and the resulting size stratification are required to better understand and predict grain-size selective processes and the river bed evolution. In this study we focus on the development of such a method to measure the size stratification in sand-gravel laboratory experiments.

Before moving to techniques measuring the size stratification in the bed, we first discuss measuring the grain-size distribution of the bed surface. An overview of earlier methods to measure the grain-size distribution of the bed surface such as sieving and grid sampling were presented by Kellerhals and Bray (1971) and wax sampling by Diplas and Sutherland (1988). These procedures are commonly used to collect samples from a gravel bed surface. Wax sampling generally is limited to coarser mixtures because of the limited penetration of liquid wax in the pores of finer mixtures. In the case of grid sampling the grains lying below the grid points constitute the sample. Therefore application of the method is limited to collecting mostly coarse sediment. The major drawback of these techniques is their time-consuming procedure for determining the grain-size distribution due to the time required for taking the samples as well as for sieve analyses.

It is worth noticing that particularly in the case of determining the grain-size distribution of the bed surface the results of the areal sampling techniques (e.g. wax or clay sampling, image analysis) are not equivalent to the outcomes of sieving. These areal sampling techniques provides areal grain-size distributions that are not equivalent to the volumetric grain-size distributions originating from sieve analysis (Kellerhals and Bray, 1971). A conversion model (e.g. Kellerhals and Bray, 1971; Diplas and Sutherland, 1988; Fraccarollo and Marion, 1995) is then needed to convert areal fraction contents into volume fraction contents.

In the past years image analysis techniques have emerged as a reliable, practical and non-destructive procedure (McEwan et al., 2000; Sime and Ferguson, 2003; Graham et al., 2005a, b, 2010). The first image processing techniques still required laborious work through manual measuring on the images, overlaying transparent grids on the image to count the grains (Adam, 1979) or manually digitizing large prints to delineate the particle edges (Ibbeken and Scheyler, 1986). The development of fully automated methods was obviously connected to the advancements in computer technology. The common automated procedures are based on detecting the edges of individual grains and determining the particle dimensions (McEwan et al., 2000; Sime and Ferguson, 2003; Graham et al., 2005a, b, 2010). Application of these techniques to a variety of grain shapes, lithotypes, texture, and sizes still needs to be assessed (Graham et al., 2005a). The limiting accuracy for the finer particles is controlled by the resolution of the image. Graham et al. (2010) remark that even when accounting for the improvement in the resolution of digital cameras, there will be constraints in the accurate identification of the edges of the smaller grains. A compromise between the minimum grain size that can be detected and the surface area covered by an image is required.

The restriction to the minimum grain size that can be detected within a certain surface area covered by the image was avoided by combining the image analysis with particle colouring (Wilcock and McArdell, 1993, 1997; Wu and Yang, 2004). In their laboratory experiments, the detection of the edges of the particles is replaced by the detection of colour. This has enabled application of image analysis to smaller grain sizes: sands and fine gravels (Wilcock and McArdell, 1993, 1997; Wu and Yang, 2004). Wilcock and McArdell $(1993,1997)$ introduced the so-called bed of many colours (BOMC) to evaluate the grain-size distribution of the bed surface. They placed a grid over the images and the colours of the grains positioned on the grid intersections were registered. Wu and Yang (2004) modified the procedure of Wilcock and McArdell $(1993,1997)$ and counted the number of grains present inside the grid cells in images taken at different times to also allow for the detection of grain mobility. An improvement in image analysis combined with particle colouring was suggested by Heays et al. (2010a, b). Their approach consists of recognizing sediment colours by setting RGB colour thresholds in order to detect a specific colour and the isolation of grain clusters based on colour. Detecting the fraction contents of the grain-size fractions as grain clusters rather than the size of individual grains allows to automatically and rapidly determine the areal fraction contents covered by each colour. Yet, the measurement of other parameters, such as particle velocity (Wu and Yang, 2004; Heays et al., 2010a, b) still requires the detection of individual or small groups of particles.

More recently, an innovative approach based on the characteristics of image pixels was developed for photographic techniques used in field campaigns. In ground-based photography (Rubin, 2004; Warrick et al., 2009) and airborne photography (Carbonneau et al., 2004, 2005; Dugdale et al., 2010), relationships were found between grain-size properties and the pixel properties. These properties, such as pixel 
brightness, are measured using approaches based on spatial statistics. Rubin (2004) presented a spatial autocorrelation method to measure the correlation between the pixel intensity of two regions of the image. The method was applied to measure subaqueously the grain-size distribution of the bed surface and vertical profiles. Images with an unknown grain-size distribution are analyzed comparing them with autocorrelation curves that are representative of a certain sediment grain size. This method used as well by Warrick et al. (2009) can be applied to a large range of grain sizes. The second approach based on spatial statistics consists in measuring the local semi-variance, which is a function of the difference in brightness and the distance between these pixels (Carbonneau et al., 2004). This second method was applied in airborne photography for the grain-size mapping of river catchment areas (Carbonneau et al., 2004, 2005; Dugdale et al., 2010). An application of the Carbonneau et al. (2005) method was used to study the evolution of braided river deposit in a flume experiment (Gardner and Ashmore, 2011).

A large variety of samplers and box corers is available to determine the size stratigraphy of a deposit formed out of cohesive sediment, whereas measuring the stratigraphy of a non-cohesive deposit has always been a challenging task as the sediment sample drops from the box corer unless the box is closed or the sample is fixed with some substance. The combination of using a box corer with sieve analysis is a common method to obtain information about the vertical variation of the grain-size distribution. Jonasson and Olausson (1966) proposed two devices with different closing mechanisms for sampling the uppermost layers of marine sediment. The method does not allow for undisturbed samples and the operation procedure together with the devices' large dimensions limit their use to field surveys. Blom et al. (2003) developed a box coring method to analyse the vertical sorting within a sand-gravel bed under laboratory conditions. The sampler is shaped as a rectangular box which is closed using a rectangular plate that is pushed obliquely into the sample. After it is closed, the box is removed from the bed. A drawback is the fact that some grains are pushed down when pushing the box into the bed and when pushing down the closing device.

In order to take samples out of non-cohesive sediment in a less disturbed way, many methods are based on pouring a liquid substance inside the pores and letting the sample solidify. In this manner the particles are attached to each other and to the sampling tool (i.e. box corer or probe pipe) creating a sample that can be lifted from the bed in one piece. Various fixing methods are used: wax coring (Sibanda et al., 2000); freezing samples by liquid nitrogen (Gomez, 1983; Pallara et al., 2006) or liquid carbon dioxide (Walkotten, 1976; Carling and Reader, 1981), and epoxy resin (Alexander et al., 2001). The resulting sample is then analysed melting the fixing substance (Walkotten, 1976; Gomez, 1983; Sibanda et al., 2000) or first cutting sections of the sample at various elevations (Carling and Reader, 1981) and sieving the sedi- ment. The sampling method presented by Marion and Fraccarollo (1997) is one of the least invasive freezing methods since the sample containers are introduced inside the flume before starting the experiment. The samples are removed and later frozen. These frozen blocks are broken in four pieces and the inner undisturbed surfaces are photographed in order to measure the vertical variation of the grain-size distribution through image analysis. The parts are later recomposed and placed back into the flume and, after thawing, the experiment is restarted. Repeating this procedure several times allowed Marion and Fraccarollo (1997) to study the evolution of the size stratification of the bed.

A final option to define the size stratification in laboratory experiments consists of removing separate layers from the deposit and subsequently analyse the grain-size distribution through sieve analysis. This was realized by means of siphoning of sand layers by Ribberink (1987) or, for a coarser sand-gravel mixture, removing the sediment layers using a spatula by Kleinhans (2005).

The objective of this paper is to present a method for measuring the size stratification of a deposit in laboratory sandgravel experiments. The method combines three of the above techniques: (1) particle colouring, (2) removal of thin sediment layers, and (3) image analysis. The image analysis is based on the approach by Heays et al. $(2010 \mathrm{a}, \mathrm{b})$. The difference lies in the fact that (a) we extend the image analysis to analysing the grain-size distribution of the substrate rather than only the bed surface, (b) we use another algorithm for colour detection, and (c) we work with the wider subset of colours of the Lab colour space. In Heays et al. (2010a, b) colour detection is based on the isolation of a limited number of colours (equal to the number of grain sizes), with a consequent loss or non-detection of pixels (e.g. darker grains due to shadows). Instead our approach is based on colour segmentation and therefore assigns a colour to each pixel in the image. The areal fraction content of a certain grainsize fraction is measured rather than measuring the individual grain sizes. This is because the stratification measurement method was developed to compare future data from flume experiments with the results of models such as the surfacebased transport model for mixed sediment (e.g. Wilcock and Crowe, 2003), and a sediment continuity model for mixed sediment (e.g. Hirano, 1971; Ribberink, 1987; Blom, 2008), which require information on the fraction content for a range of grain sizes at the bed surface and substrate.

\section{The size stratification measurement method}

In this section we present the three steps in the new method to measure the size stratification of a coloured sand-gravel deposit: (1) particle colouring, (2) removal of thin sediment layers, and (3) image analysis. The deposit used to assess the applicability of the size stratification measurement method 


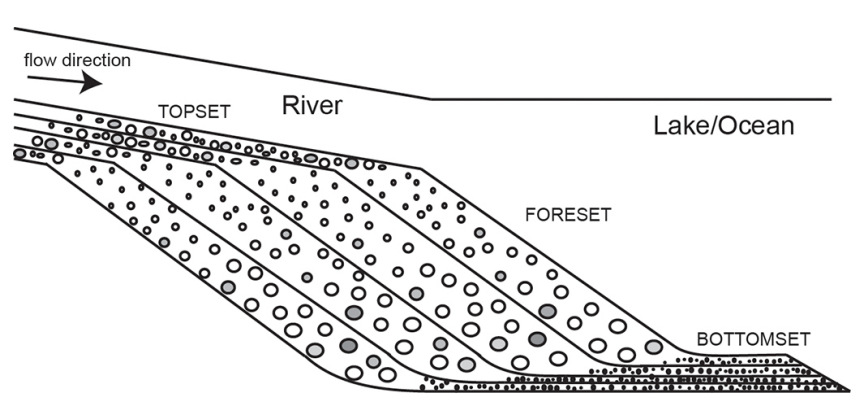

Figure 1. Schematic of a Gilbert-type delta (after Gilbert, 1890).

is a Gilbert or foreset-dominated delta formed in laboratory experiments (Chavarrías et al., 2013, 2014).

The stratigraphy of foreset-dominated deltas differs from the one of the well-known topset-dominated deltas (Edmonds et al., 2011). Foreset-dominated deltas are deltas that are governed by depositional processes over the delta front. The foreset deposit, comprises the larger part of the deposit. In topset-dominated deltas sediment deposition mainly takes place on the delta top or fluvial reach. This fluvial reach consists of distributary channels and levees, river mouth bars and inter-distributary bays (Edmonds et al., 2011).

A Gilbert delta (Fig. 1) is formed where (a) a river flows into a deep basin such as a lake or an ocean, and (b) sediment deposition is dominated by avalanches over its steep slip face. Its stratigraphy consists of three parts (Gilbert, 1885): (a) a topset that forms as a result of deposition over the fluvial reach, (b) a foreset that forms as a result of avalanches of grains (i.e. grain flows) over a steep lee face, and (c) a bottomset that forms out of fine sediment that deposits downstream from the foreset as a result of grain fall. The avalanches down the steep slope of the delta front typically result in an upward fining profile. Sediment transport over the fluvial reach results in the delivery of sediment to the delta brinkpoint. Due to the sudden expansion of the flow the sediment deposits over the brinkpoint forming a wedge. When the angle of repose is exceeded the sediment avalanches down the delta front. These avalanches makes the delta prograde. The delta progradation leads to the lengthening of the fluvial reach and the decrease of the river bed slope. This leads to aggradation over the fluvial reach and a gradual decrease in the delta progradation rate. The rate at which the delta progades is affected by sediment supply or sea level changes (Postma, 1995).

In a drained flume areal images of the Gilbert delta were taken at different elevations of its deposit to determine its size stratification. These elevations correspond to the top of layers of $1 \mathrm{~cm}$ thickness that were removed one by one using a wet vacuum cleaner. The grain-size distribution of the surface of each sediment layer is analysed through image analysis. We compare the results of the image analysis to the results of sieve analysis to assess the image analysis method. The required steps are explained in the next few sections.

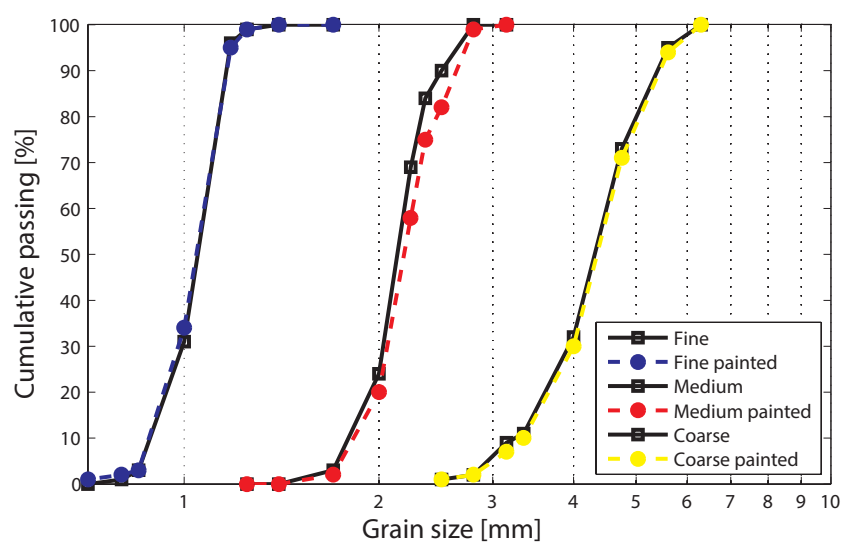

Figure 2. Grain-size distribution of the original sediment (black lines) and the coloured sediment (blue, red and yellow lines).

\subsection{Particle colouring}

The sand-gravel deposit was composed of three well-sorted grain-size fractions within the range of coarse sand to fine gravel. We considered the fractions to have negligible overlap since sieve analysis indicated only a slight overlap of 1-2\% between medium fraction 2 and coarse 3 (Fig. 2). The grain-size fractions were non-overlapping to facilitate the sieve analysis, which is required to evaluate the image analysis technique. The original sediment mixture is composed of a fine size fraction $\left(D_{50,1}=1.05 \mathrm{~mm}\right)$, a medium size fraction $\left(D_{50,2}=2.13 \mathrm{~mm}\right)$ and a coarse size fraction $\left(D_{50,3}=4.32 \mathrm{~mm}\right)$.

The grains were coloured with a common wall paint using a concrete mixer. Each size fraction was coloured twice to obtain a resistant and bright colour and to fully coat the grains with paint. Sieve analysis was performed to determine the grain-size distribution of the painted sediment. Only little difference between the grain-size distribution of the original and the painted grain-size fractions is found (Fig. 2). The median grain sizes of the painted fractions are $D_{50,1 P}=1.04 \mathrm{~mm}$ (fine fraction, blue), $D_{50,2 P}=2.19 \mathrm{~mm}$ (medium fraction, red) and $D_{50,3 P}=4.36 \mathrm{~mm}$ (coarse fraction, yellow).

The densities of the original and the painted grain-size fractions were measured using a hydrostatic balance. We noticed a slight decrease in mass density for the three grain-size fractions due to the painting, which was considered acceptable. The densities of the original sediment are $2614 \mathrm{~kg} \mathrm{~m}^{-3}$ (fine), $2614 \mathrm{~kg} \mathrm{~m}^{-3}$ (medium), $2600 \mathrm{~kg} \mathrm{~m}^{-3}$ (coarse). The densities of the painted fractions are $2590 \mathrm{~kg} \mathrm{~m}^{-3}$ (fine), $2564 \mathrm{~kg} \mathrm{~m}^{-3}$ (medium), $2549 \mathrm{~kg} \mathrm{~m}^{-3}$ (coarse).

\subsection{Removal of sediment layers}

The water was drained from the flume once the experiment was finished. The sampling procedure was started when the bed was dry. Two metal sheets with a thickness of $2 \mathrm{~mm}$, a 


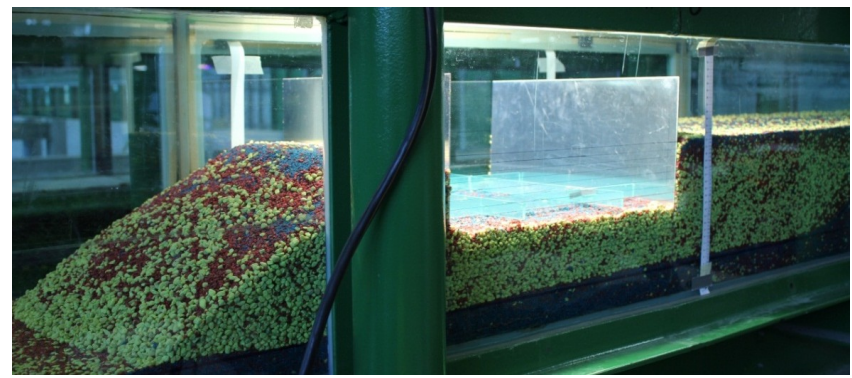

Figure 3. Experimental set-up. Side view of the delta progradation experiment during the removal of sediment layers and sampling for sieving purposes.

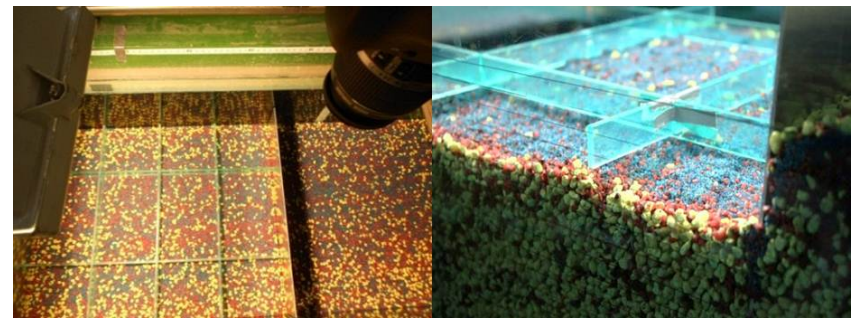

Figure 4. Set-up for image analysis and sampling: top view of the sampling area with the sampling grid placed on the bed surface and the metal sheets inserted into the bed. The Plexiglas ${ }^{\circledR}$ grid and sampling per grid cell is only needed for the assessment phase of the image analysis method. Once the conversion method is clear, the sampling grid is no longer required and an entire thin sediment layer can be removed at once without using the grid.

width of $0.39 \mathrm{~m}$ and a height of $0.30 \mathrm{~m}$ were driven along the edges of the sampling area into the delta deposit to support the sediment faces while thin sediment layers in between the metal sheets were removed (Fig. 3).

A rigid Plexiglas ${ }^{\circledR}$ sampling grid was placed on the bed surface and was used to divide the reach into patches (Fig. 4). The reach was subdivided to enable a comparison of the local grain-size distribution determined through image analysis with the one determined through sieving. The Plexiglas ${ }^{\circledR}$ grid and the sieve analysis are only required when assessing the image analysis procedure. Please note that once the method is validated, the sampling grid is no longer required and an entire sediment layer can be removed at once without using the grid. A grid with a vertical grid size of $1 \mathrm{~cm}$ was indicated on the glass side walls of the flume as an aid to removing the $1 \mathrm{~cm}$ sediment layers. We took areal images of the deposit within each grid cell. After this a $1 \mathrm{~cm}$ sediment layer was removed from each grid cell and later sieved. Subsequently the camera position was lowered by $1 \mathrm{~cm}$ and the full procedure was repeated until reaching the parent material of the initial bed. We excluded the grid cells bordering the metal sheets from the analysis as the sediment in that area may have been disturbed by driving the metal sheets into the bed.

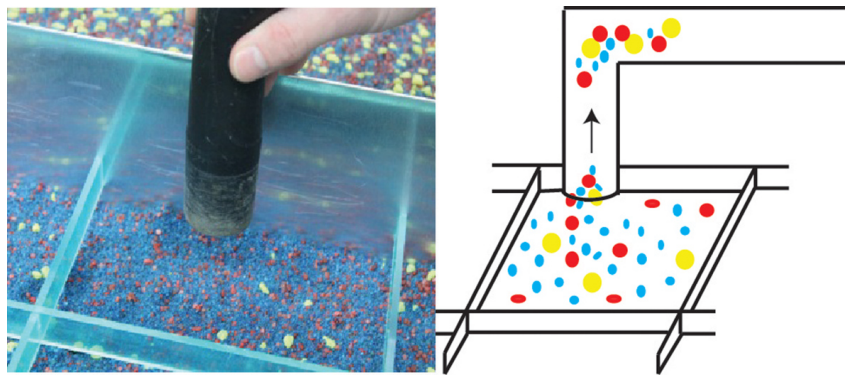

Figure 5. Nozzle of vacuum cleaner close to the bed surface removing the $1 \mathrm{~cm}$ sediment layers.

The $1 \mathrm{~cm}$ sediment layers were removed using a wet vacuum cleaner (Fig. 5). The vacuum nozzle was placed at a certain distance from the surface of the deposit to extract the sediment. The sediment extracted from each grid cell was collected from the vacuum cleaner cylinder, and stored for sieve analysis. The cylinder was created with a filter net having a mesh smaller than the finest fraction. This was done to reduce the time required to collect the sediment inside the vacuum cleaner. The sampling with the vacuum cleaner was not size-selective and did not show a preference for the fine fractions. The fact that the sediment was painted helped us to verify the removal of all size fractions. For instance, the coarser yellow particles were clearly distinguishable and it was visible when they were removed by the vacuum cleaner together with the finer fractions. The vacuum cleaner was used passing over the same area several times until the $1 \mathrm{~cm}$ thick layer was removed. This helped us to collect all fractions from that layer.

In case continuation of an experiment is required, the removed sediment patches can be placed back separately at the original elevations of the deposit after sampling. It is clear that this option is feasible only when sampling restricted areas of a deposit.

\subsection{Image analysis}

The areal images taken during the experiments are processed with an image analysis algorithm that is written using Matlab. The code is based on colour segmentation and provides the areal fraction content of a surface area covered by a certain colour, i.e. a certain grain size (Fig. 6). Colour segmentation indicates the division of all the pixels present in an image into a limited number of imposed colour groups (i.e. clusters). The camera lens used, the Canon EF-S 18-55 mm IS f/3.5-5.6 wide-angle to mild telephoto, has very small geometric distortions. The barrel distortion is $3.35 \%$ at a focal length of $18 \mathrm{~mm}$, which means that at the applied focal length of $55 \mathrm{~mm}$ the barrel distortion is lower than $3.35 \%$. The pincushion distortion at a focal length of $55 \mathrm{~mm}$ is $0.389 \%$. For these reasons we decided not to correct the images for geometric distortions. 


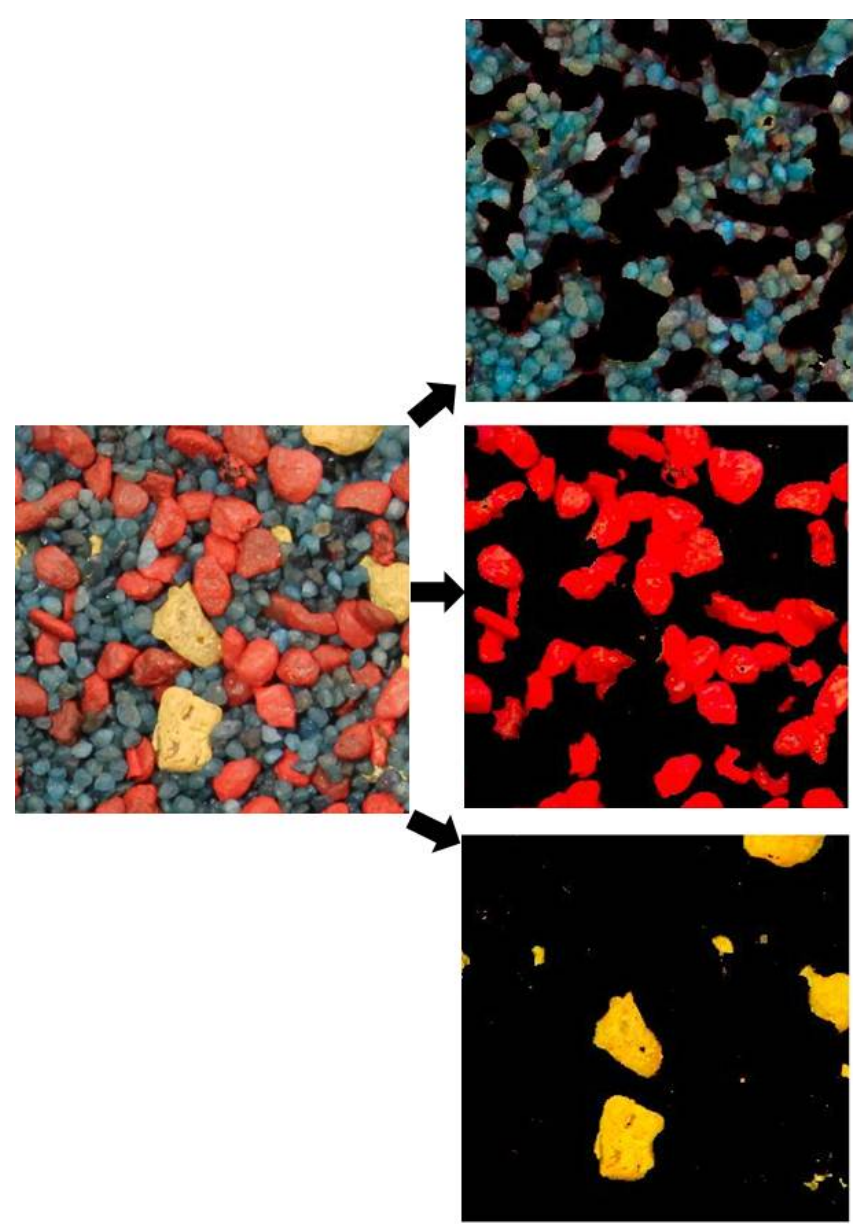

Figure 6. Colour segmentation for the three grain-size fractions.

A preparatory step in the procedure concerns cropping of the images and the detection of cluster centre values. Each cluster centre corresponds to a colour (i.e. grain size) and indicates the mean values of its colour coordinates in colour space. In other words, if we have $N$ grain-size fractions, we define $N$ cluster centres. These cluster centres are determined analysing a limited number of images (in our case $5 \%$ of all images). We consider images in which the presence of each colour is at least higher than $10 \%$ to obtain appropriate cluster centres, thus allowing for a proper colour segmentation. The colours are detected automatically using $k$-means clustering while imposing the number of colours; $k$-means clustering (MacQueen, 1967) is a partitioning method aimed at dividing a set of objects into groups (i.e. clusters) based on their attributes. Each object has a location in space. The distance from this location to a representative point of the cluster (i.e. cluster centre) is used to define to which group the object belongs. Using an iterative algorithm the objects are moved between the clusters until the sum of distances from each object to its cluster centroid is minimized. Although the method can be applied to a wide range of cases, here we use it to divide a set of pixels into colour groups to determine an average value for each colour (i.e. cluster centre). The colour segmentation is carried out in the Lab colour space. The Lab colour space is a colour model as the RGB, and is composed of three dimensions. In the case of RGB the dimensions are given by red, green and blue values. A colour results from the combination of the percentages of red, green, and blue values. The Lab colour space combines the luminosity dimension $L$ and the colour (so chromatic) dimensions $a$ and $b$. This means that the colour information is in the $a$ and $b$ dimensions. The parameter $a$ indicates how the colour plots along the red-green axis, and $b$ indicates how it plots along the blue-yellow axis.

The procedure to determine appropriate cluster centres based on a selection of images is summarized as follows:

1. image reading;

2. colour saturation (Zaman, 2011);

3. image conversion from RGB colour space to Lab colour space;

4. colour classification by $k$-means clustering to obtain the cluster centres;

5. taking the average of the cluster centres.

Below these steps are explained in more detail:

1. The RGB images chosen to determine appropriate cluster centres are loaded.

2. Colour saturation enhances the image colours and gives more uniformity to the different tones of the grains.

3. The RGB values of each pixel are converted into Lab colour space values. In the Lab colour space each pixel includes a combination of three indices consisting of a luminosity value $L$ and two chromaticity values $a$ and $b$ that delineate the colours.

4. The segmentation is based on $k$-means clustering. The function checks the colours by means of the $a$ and $b$ values of the image and it separates them into the different clusters comparing their values. The cluster centre values consist of the mean $a$ and $b$ values for each cluster.

5. The cluster centre values of the selection of images are averaged using the $k$-means function to compute one mean centre value per cluster. Please note that in this case the $k$-means algorithm is applied to average the cluster centre values of the images instead of clustering the pixels of the images as done in point 4 .

Subsequently, in all images the colours are segmented imposing the cluster centre values determined under step 5 . This a priori setting of the cluster values leads to a much faster computation during the image analysis and most importantly to a more robust colour segmentation. More specifically, the 
colour segmentation based on forced cluster centres shows good results even for cases in which when one of the colours has an areal fraction smaller than $10 \%$, whereas $k$-means clustering results in an incorrect clustering of the colours under these conditions. The procedure to determine the areal fraction content of each colour for all images based on the forced cluster centres is summarized as follows:

1. initialization of the cluster centre variables;

2. image reading;

3. colour saturation (Zaman, 2011);

4. image conversion from RGB colour space to Lab colour space;

5. calculation of the distances of $a$ and $b$ coordinates to the cluster centres;

6. colour segmentation defining the minimum distances;

7. calculation of areal fraction content covered by each colour.

Below these steps are explained in more detail:

1. The cluster centre values of the $N$ colours, defined in steps 1-5, are set as input parameters.

2. The RGB images are loaded and read.

3. Colour saturation is performed to render the image colours uniform.

4. The RGB values of each pixel are converted into the Lab colour space values.

5. The chromaticity values $a$ and $b$ are used to classify the colours. The distances between the $a$ and $b$ coordinates and the cluster centres of the $N$ colours (step 6) are calculated for each pixel in the image (i.e. for each pixel there are $N$ values for the distance to each cluster centre).

6. Among the $N$ distances calculated, a minimum distance is identified. The value of the minimum distance determines in which cluster (i.e. to which colour) every pixel is allocated (Fig. 7).

7. The pixels within each cluster are counted and the areal fraction covered by each colour in the image is computed.

The image analysis algorithm proposed here assigns a cluster to $100 \%$ of the pixels of the image. In Heays et al. $(2010 \mathrm{a}, \mathrm{b})$ colour detection is based on the isolation of each colour, with consequently a loss or non-detection of some pixels due to shadows in the image or particles with a darker colour. For these reasons their maximum surface detection reached for the images is $91 \%$. Moreover, rather than

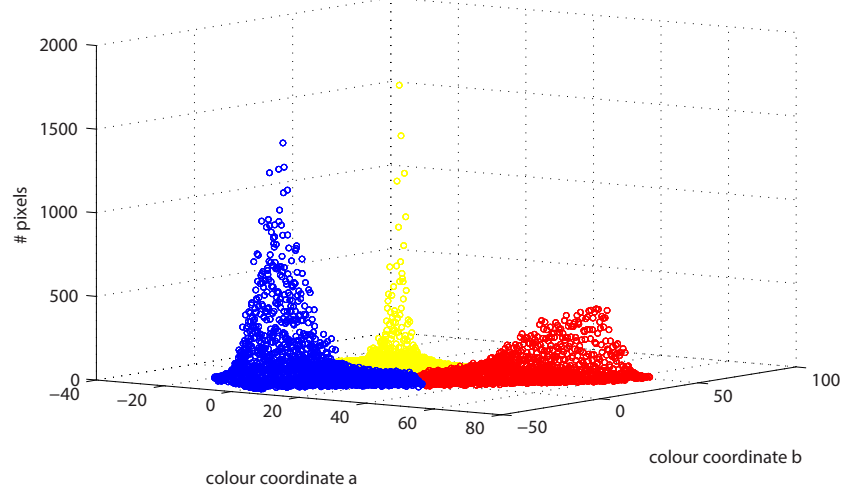

Figure 7. Three-dimensional view of the $a$ and $b$ domain. Example for one image. Clusters segmented in the three colours: blue, red and yellow. The position of each dot in the $a$ and $b$ plane is related to the colour and its vertical position indicates the number of all pixels with the same colour coordinates.

working in the RGB colour space like Heays et al. (2010a, b), we work in the wider subset of colours of the Lab colour space which includes all perceivable colours. Our algorithm considers the two-dimensional space composed of the two chromaticity values $a$ and $b$. Using the Lab colour space instead of the three-dimensional RGB colour space results in a shorter computational time for the algorithm.

The image analysis procedure presented here was applied to measure the size stratification of a coloured sand-gravel deposit. Figure 8 shows a schematic of the method. Images of the deposit are taken at various elevations and each image is processed with the above described algorithm in order to obtain the areal fraction content covered by each colour (i.e. grain size). Before discussing the results of applying the method to the Gilbert delta (Sect. 4), the stratification measurement method will be assessed in the next section.

\section{Assessment of the size stratification measurement method}

The stratification measurement method was assessed in two sets of experiments conducted at the Environmental Fluid Mechanics Laboratory of Delft University of Technology. The image analysis technique was optimized and tested in an image analysis experiment (Fig. 9). Subsequently, the stratification measurement method was applied to a Gilbert delta progradation experiment. The latter analysis will be described in Sect. 4.

\subsection{Image analysis experiment}

The image analysis experiment was performed to determine the best settings to take the images and to develop, optimize, and test the image processing algorithm. Once the best settings (e.g. lights, camera settings) were chosen, images were 


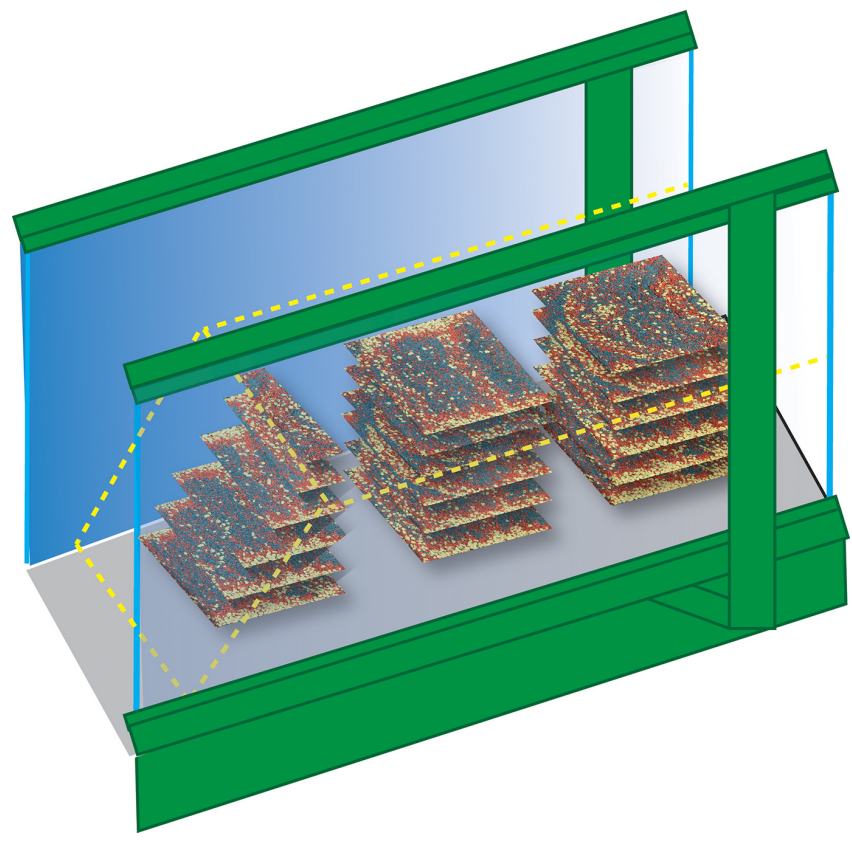

Figure 8. Application of the image analysis technique to stratigraphy measurements. Schematic of size stratification of a deposit evaluated with image analysis.

taken of the same area of the bed for different water depths. The experiment was realized in a rectangular pool in which a wooden box of $2 \mathrm{~m}$ length, $1.04 \mathrm{~m}$ width, and $0.60 \mathrm{~m}$ height was placed. The box was divided into 12 compartments with vertical wooden panels and filled with sediment (Fig. 9). One compartment was left empty to place the water pump for varying the water depth in the pool. The experimental set-up was designed to test also other measurement techniques and patches 1-3 and 5-9 were used for testing the image analysis procedure.

We used the same three well-sorted non-overlapping grain-size fractions as described in Sect. 2.1. For some patches we used the finest fraction in its natural colour to evaluate if the image analysis can be applied to unpainted fines. This would avoid painting and potential stickiness of large quantities of relatively fine sediment.

The compartments used to test the image analysis were filled with different mixtures, that is, different volume fraction contents of the three grain-size fractions and by different colour combinations (Fig. 9). The colour combinations are (a) blue for the fine size fraction, red for the medium size fraction, and yellow for the coarse size fraction (patches 1 , 3,5 ), and (b) natural colour for the fine size fraction, red for the medium size fraction, and blue for the coarse size fraction (patches 2, 6). In the remaining compartments a single size fraction was present on the surface, that is, one colour (patches 7-9). It is important to note that in the compartments filled with sediment mixtures (patches $1-3,5,6$ ), it was not possible to exactly reproduce the foreseen areal frac-

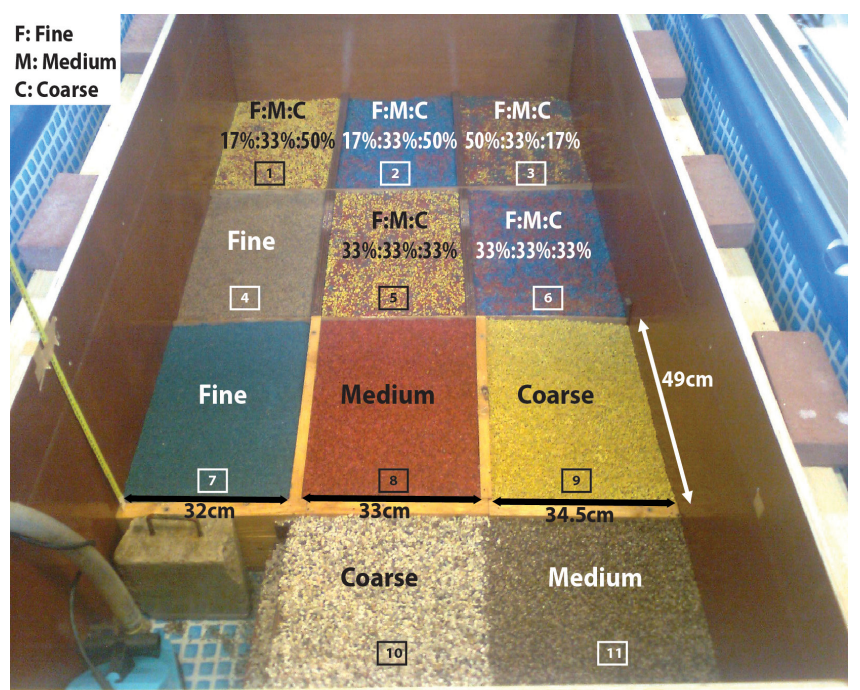

Figure 9. Settings of the image analysis experiment. Grain-size distribution within the compartments, compartment geometry and numbers.

tion content of each size fraction on the bed surface. This is because, while pouring the sediment the finer grains, on average, deposit at lower elevations due to kinetic sieving.

The camera used in the experiment was a Canon EOS 550D, an 18.0 megapixel digital single-lens reflex camera with a CMOS sensor. It has a maximum resolution of $5184 \times 3456$ and $3: 2$ aspect ratio. Images were stored in colour JPEG format and taken in Live View mode. The camera was used with the Canon EF-S $18-55 \mathrm{~mm}$ IS f/3.5-5.6 wide-angle to mild telephoto zoom lens. Tests were carried out to assess the camera lens and the proper camera height. The distance between the camera and the bed surface was set to $50 \mathrm{~cm}$. The focal length for the camera lens was $55 \mathrm{~mm}$. All pictures were taken with the auto exposure program (PAE) and without flash and the lens was used with the image stabilizer function (IS), which automatically corrects for vibrations of the photo camera.

In order to apply this method to submerged beds, a Plexiglas ${ }^{\circledR}$ plate was placed on the water surface to reduce reflection and refraction caused by water surface fluctuations (Wu and Yang, 2004). We used four flood lights to illuminate the bed surface. The lights were switched on at least $20 \mathrm{~min}$ before taking the pictures to provide a steady illumination and to avoid reflections on the Plexiglas ${ }^{\circledR}$ plate from the different sources of light in the laboratory. In this way there was as little influence as possible from (naturally varying) natural light from outside.

In the image analysis experiment the area of the bed surface covered by the pictures was ca. $18 \mathrm{~cm} \times 12 \mathrm{~cm}$ and the pixel resolution was $0.03 \mathrm{~mm}$. The upper limit of the tested pixel resolution was $0.1 \mathrm{~mm}$ (pixel dimension). For this 


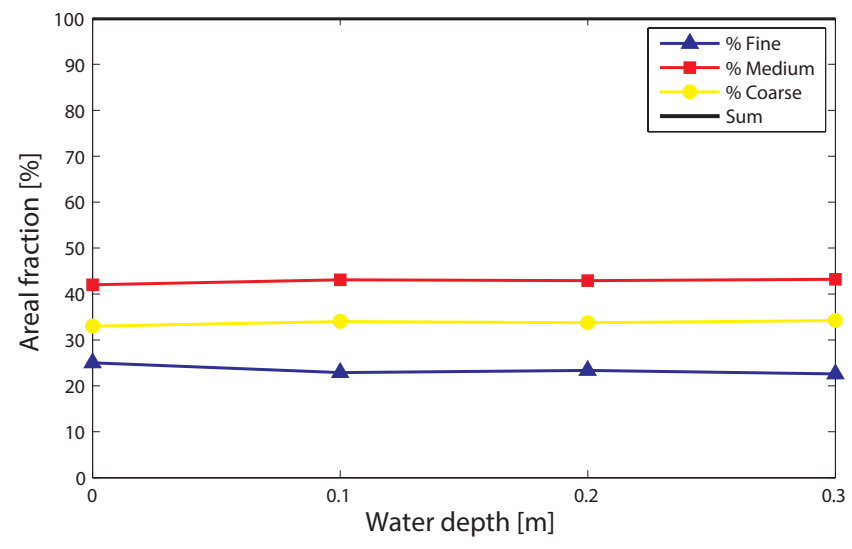

Figure 10. Example of the effect of water depth on the areal fraction in patch 5. The data represent the areal fraction of the three size fractions for different water depths obtained through processing of images covering the same surface area.

resolution we still obtain a correct segmentation of colours in the image processing. With the camera settings for this maximum pixel resolution, we can cover a bed surface area of ca. $60 \mathrm{~cm} \times 40 \mathrm{~cm}$ in one image. A higher camera resolution would enable covering even bigger bed surface areas.

Mixtures composed of a blue fine fraction appeared to be segmented more accurately than the ones in which the fines were of a natural colour. The remaining part of this paper considers the colour combination blue (fine), red (medium), and yellow (coarse).

The camera was mounted on a carriage that was controlled by an operator and a computer to move the camera over the pool. The camera coordinates were registered to be able to reposition the camera at the same location to take pictures for different water depths. In this way we obtained images of equal surface areas for water depths equal to $0.0,0.1,0.2$, and $0.3 \mathrm{~m}$. The water depth was increased by inserting a hose in the space between the wooden box wall and the swimming pool wall in order to have negligible flow velocities to not disturb the bed surface. The water depth appeared to not significantly affect the resulting grain-size distribution (Fig. 10). Yet, images for the submerged cases showed somewhat better results compared to the unsubmerged case, since in the presence of water the colours were more saturated. Certain images taken of an unsubmerged bed showed some white pixels in the segmented image for the colour blue, which are caused by reflections due to water drops (Fig. 11). For this reason it is advised to wait for the sediment to dry when taking pictures of an unsubmerged bed surface, which can require a few hours.

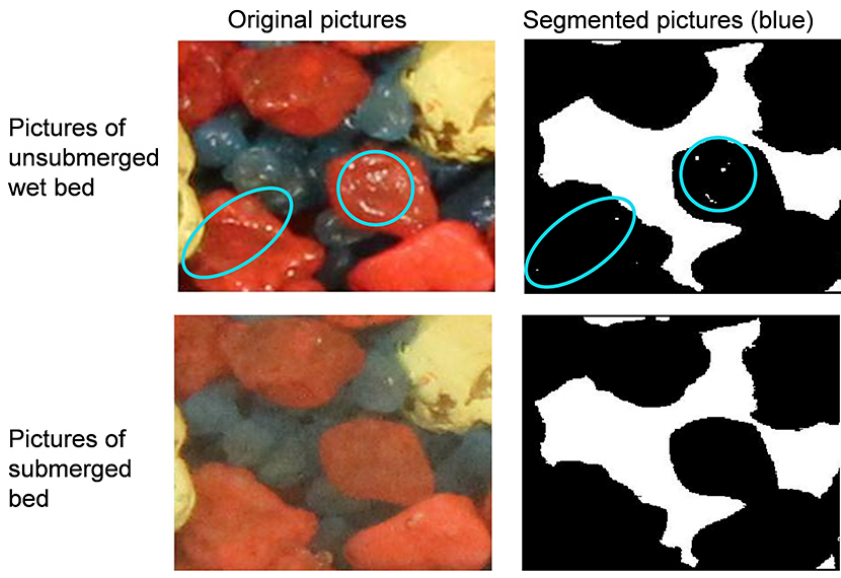

Figure 11. Origin of white pixels in images of an unsubmerged bed; left: original images; right: binary image of the segmented image for the colour blue; at the top: images for an unsubmerged bed; at the bottom: images for a submerged bed. The extra white pixels in the unsubmerged pictures, indicated with blue circles, are due to reflections of water drops.

\subsection{Accuracy of the size stratification measurements}

The sources of inaccuracies in the size stratification measurement technique are due to a combination of inaccuracies in the (1) particle colouring, (2) removal of thin sediment layers, and (3) image analysis.

An imperfect or thin coating of the paint over the grains showing the original colour of the sediment may lead to inaccuracies in the colour segmentation. We have reduced this effect by colouring the grains twice. Another issue is due to the potential stickiness of grains which can be almost completely avoided by shaking and mixing the sediment several times while the paint is drying. Yet, the medium size fraction consisted of some flat grains which appeared to be difficult to separate during the shaking procedure (Fig. 2). An important aspect for the colour segmentation is the choice of the combination of the colours. For a proper colour segmentation it is essential to choose colours that are as different as possible. This means as distant as possible in the applied colour space. Also the original colour of the sediment plays a role and can cause the resulting colour of the painted grains to be slightly different from the paint colour.

The inaccuracies in the removal of the sediment layers are mostly related to using the wet vacuum cleaner. As discussed before the wet vacuum cleaner did not appear to be grain-size selective. A source of inaccuracy in the sediment removal is the precision in removing layers of $1 \mathrm{~cm}$ thickness. A vertical grid was created on the side walls of the flume to aid the estimation of the layer thickness. Based on our observations the inaccuracy in thickness of the sediment layers was between 1 to $2 \mathrm{~mm}$. The imprecision is caused by an oblique view of the grid due to the position of the operator or the distortion due to the glass of the side walls. Another reason for 


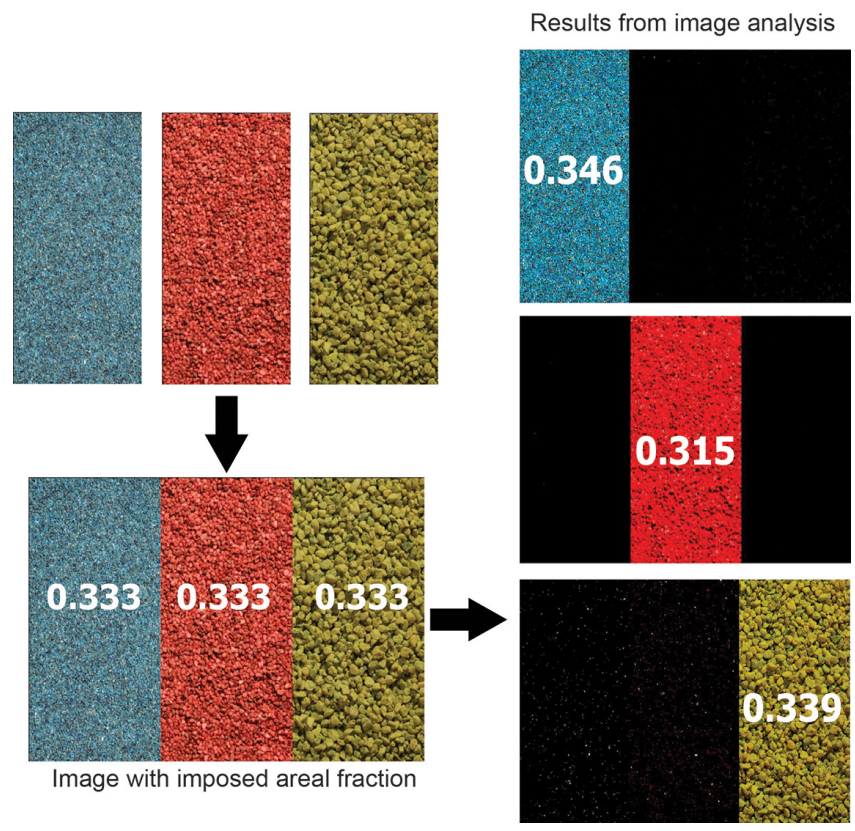

Figure 12. Accuracy test; left: Image with imposed areal fraction content created by combining three images of uniform sediment of the same pixel area; right: segmented images and mean areal fraction contents resulting from the image processing.

inaccuracy in the layer thickness is due to varying the elevation of the mouth of the wet vacuum cleaner above the bed surface. Please note that these inaccuracies in the thickness do not have a cumulative effect while removing more and more sediment layers.

The fact that there are instrinsic differences between areal and volumetric estimates of the grain-size distribution has been recognized since the earliest methods were presented (Kellerhals and Bray, 1971; Adams, 1979; Ibbeken and Schleyer, 1986) and later examined in depth in more recent studies (McEwan et al., 2000; Sime and Ferguson, 2003; Graham et al., 2005a,b; Graham et al., 2010). The main bias is related to the fact that the geometry of three-dimensional grains is estimated by a two-dimensional image (Sime and Ferguson, 2003). Graham et al. (2005b) referred to this error as the spatial distortion given by the lens projecting a three-dimensional object into a two-dimensional image. The errors described in the work of Graham et al. (2005b) are related to the image analysis technique and to the arrangement of the grains. In the case of image analysis based on particle edge detection the errors are related to the capability to detect and measure the sediment particles (Graham et al., 2005b). These inaccuracies are encountered for instance in (a) the detection of edges of a single grain, (b) the distinction between voids and parts of grains in the shadow, and (c) the estimation of the grain axes dimensions. With respect to the errors related to the arrangement of the exposed grains, Graham et al. (2010) distinguished the following three causes of errors: partial burying of grains, overlapping of grains (imbrication), and grains appearing smaller when viewed from an angle (foreshortening). This generally results in the underestimation of the correct diameter of individual grains (Graham et al., 2010).

The image analysis method proposed here is accompanied by inaccuracies mostly related to colour segmentation. Verifying the proficiency of the image processing in the colour segmentation is not straightforward. First, the size of the finest grain-size fraction used in our experiment prevents the use of validation methods as "paint and pick" or the manual digitation used in other studies to verify the results of the image analysis (e.g. Graham et al., 2005b; Sime and Ferguson, 2003). Secondly, in the image analysis experiment it was difficult to reproduce the foreseen grain-size distribution on the bed surface as, due to kinematic sieving, in the pouring and installing process the fines tend to find lower elevations than the coarser ones.

As an alternative, we have created an image with an imposed grain-size distribution and analyse the results of the colour segmentation. The image combines the images of three areas of equal pixel size, each with a uniform grain size (colour). Each image was taken of a compartment filled with only one grain-size fraction. This means that the imposed areal fraction content equals 0.333 for each colour. The results after repeating the colour segmentation on several images show average areal fractions of 0.346 for the colour blue, 0.315 for the colour red, and 0.339 for the colour yellow (Fig. 12). The inaccuracy of $1-2 \%$ originates from an incorrect colour segmentation for shadow-rich grains. These pixels are then incorrectly clustered with a darker colour. For example, when zooming in on the images we noticed that few red particles that suffer from shadows due to partial burial underneath other grains are recognized as blue in the colour segmentation (Fig. 13).

A final source of inaccuracies is observed when the grains show a bright colour tone due to light reflections or in some cases by a defect coating of the paint showing the original often lighter colour of the sediment. Despite these inaccuracies sources the maximum inaccuracy in the image analysis has appeared to be only 0.02 (2\%) of the measured segmented area. This can be considered to be a relatively high level of accuracy in determining the grain-size distribution. Overall, the stratification measurement method, despite the sum of inaccuracies in particle colouring, sediment removal, and image analysis, results in a significantly smaller inaccuracy in measuring the size stratification of a deposit than common box corers.

\section{Size stratification measurements}

In this section we describe the application of the stratification measurement method to two Gilbert delta experiments. The size stratification within the deposit measured through image 

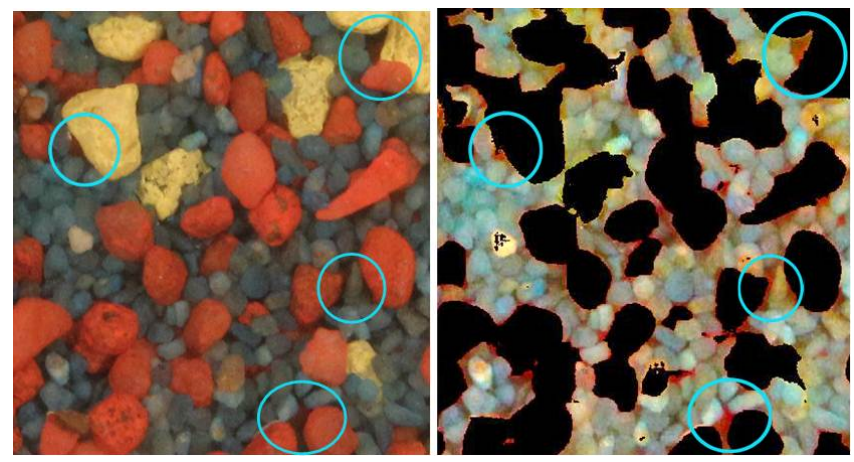

Figure 13. Example of partially buried and therefore shadow-rich red grains, indicated with blue circles, recognized as blue in the colour segmentation; left: original image of a submerged bed surface; right: corresponding segmented image for the blue colour. Please note that the blue colour in the segmented image was lightened to better illustrate the incorrect segmentation of some red grains.

analysis is compared to the one found through sieve analysis. We apply an existing conversion model (Parker, 1991a, b) that converts the areal fraction contents found through image analysis into volumetric fraction contents.

\subsection{The Gilbert delta experiment}

Laboratory experiments on the progradation of a Gilbert or foreset-dominated delta (Chavarrías et al., 2013, 2014) are used to assess the applicability of the stratification measurement method. In this paper we consider the data related to Experiments 1 and 2 (called E1 and E2) which are conducted with a constant base level, similar to Experiment I in Chavarrías et al. $(2013,2014)$. The experiments were conducted using the same initial and boundary conditions. The only difference among the two is the geometry of the resulting deltas. The length of the delta in Experiment E1 was about $1.4 \mathrm{~m}$ and in Experiment E2 was about $1.2 \mathrm{~m}$. In the experiments the sediment transported over the brinkpoint was too coarse to form a bottomset and deposited over the foreset through avalanches (grain flows). The tests were conducted with the same three sediment fractions as used in the image analysis experiment (Fig. 2) with the colour combination blue (fine), red (medium), and yellow (coarse). The flume length was $14 \mathrm{~m}$, the flume height $0.45 \mathrm{~m}$, and the flume width $0.40 \mathrm{~m}$. At the upstream end of the flume the water discharge $\left(13 \mathrm{~L} \mathrm{~s}^{-1}\right)$ was maintained constant. The downstream water level was maintained constant and created a flow depth over the brinkpoint equal to $0.06 \mathrm{~m}$, resulting in a flow velocity of $0.54 \mathrm{~m} \mathrm{~s}^{-1}$ over the brinkpoint. The sediment was fed at a constant rate of $280 \mathrm{~g} \mathrm{~min}^{-1}$ using a feeder, which was located at $2.5 \mathrm{~m}$ upstream from the initial delta brinkpoint. The volume fraction content in the sediment mixture fed to the flume as well as the one constituting the initial bed were 0.50 (fine), 0.35 (medium) and 0.15 (coarse). After the formation
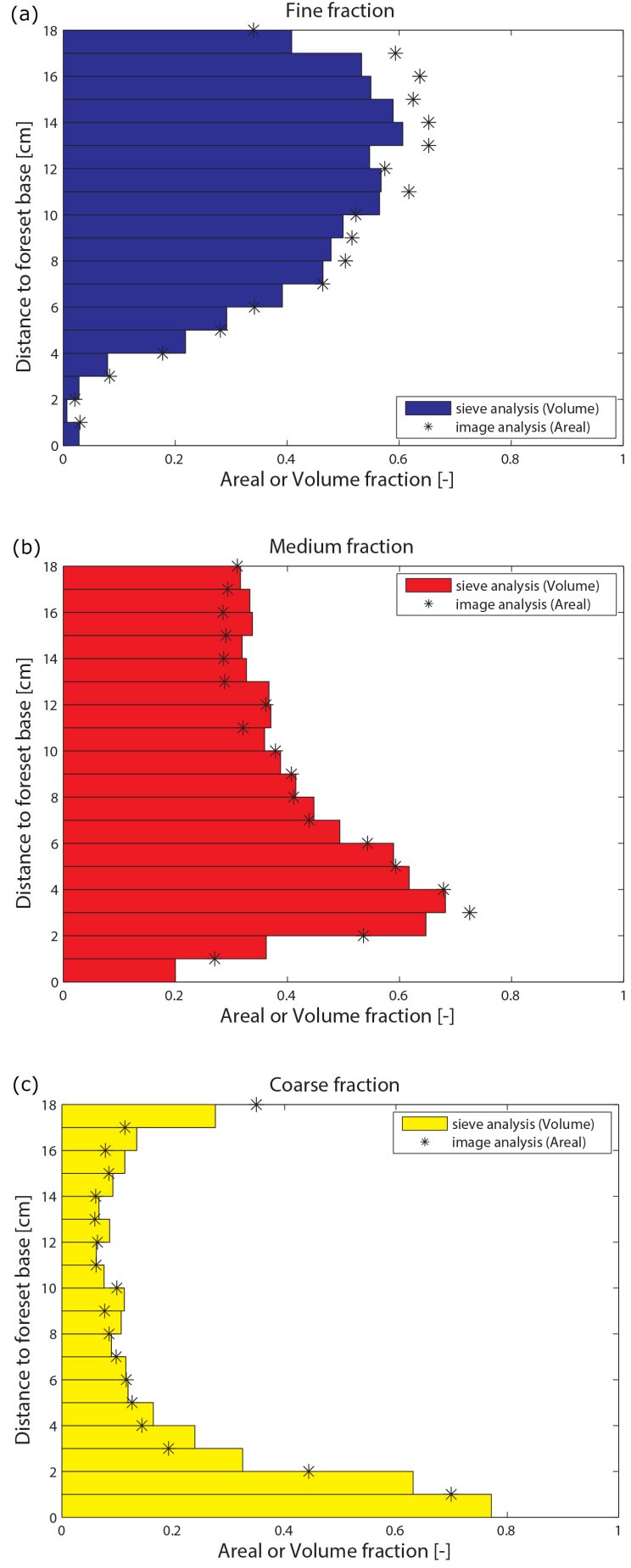

Figure 14. Vertical profiles of (1) the measured volumetric fraction contents measured through sieve analysis and (2) the areal fraction contents determined through image analysis for each grain-size fraction: (a) fine, (b) medium, and (c) coarse for Experiment E1, centre sample.

of a delta deposit, the experiment was stopped and the water was carefully drained from the flume. 


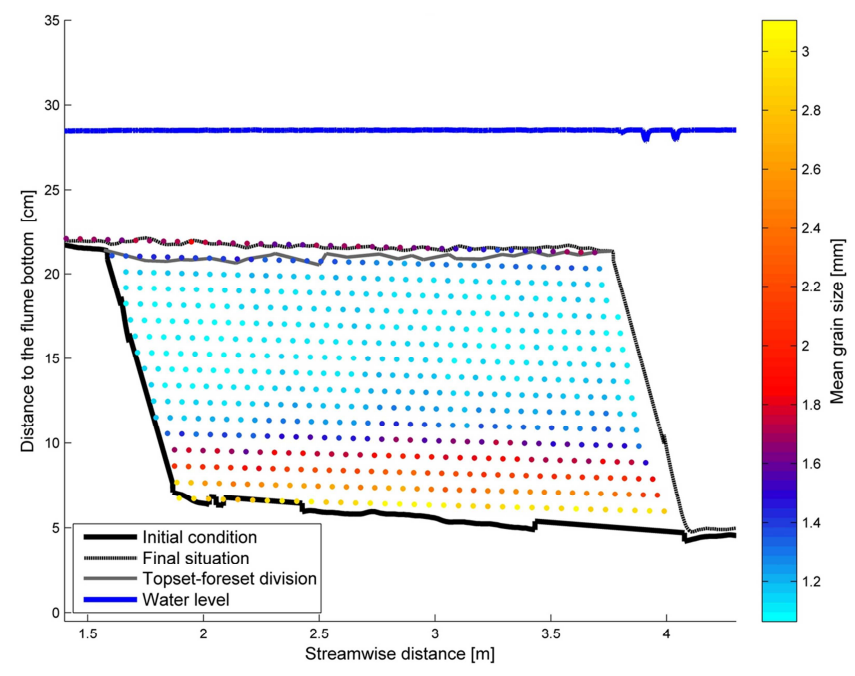

Figure 15. Size stratification within a Gilbert delta deposit measured using the stratigraphy measurement technique proposed in this paper. The colours indicate the local mean grain size based on areal fraction content.

\subsection{The Gilbert delta size stratification}

The deposits of the Gilbert deltas were analysed following the stratification measurement method described in Sect. 2.2, and using the Plexiglas ${ }^{\circledR}$ sampling grid to compare the results of the image analysis to the ones from sieve analysis. In this analysis two parts of the deposits are considered: one location at the centre of the flume and one location at the side, close to the flume walls. The two grid cells, and therefore the image, cover an area of about $24 \mathrm{~cm}$ by $18 \mathrm{~cm}$ (centre) and $24 \mathrm{~cm}$ by $11 \mathrm{~cm}$ (side). The height of the delta deposit (i.e. the distance from the top of the topset to the foreset base) was about $18 \mathrm{~cm}$ in Experiment E1 and therefore consisted of 18 sediment layers. The height of the delta deposit in Experiment E2 was about $16 \mathrm{~cm}$, with consequently 16 layers. A coarser mixture was found at the side areas, which was due to a slightly parabolic front of the delta.

In Fig. 14 the results on the areal fraction contents based on the image analysis are indicated at the elevation corresponding to the top of a specific sediment layer. The volume fraction contents resulting from sieve analysis are represented by bars covering some vertical distance, as the data represent the volume fractions of each grain size within a certain layer. Before we consider the differences between the results of the two methods, we can see that the trends of the areal fraction contents determined through image analysis and the volumetric fractions due to sieve analysis are similar. A topset is present within the vertical range of 17 to $18 \mathrm{~cm}$ from the foreset base. The upper layer of the topset is characterized by a mobile armour layer (e.g. Fig. 14c). The topset appears to be coarser than the upper part of the foreset. Below the topset we find the foreset, which is the main part of the delta deposit. It is characterized by a upward fining profile (Fig. 14a).

Let us now consider the differences between the image analysis and sieving methods. Please note how the mobile armour layer (i.e. the top layer of the topset) is more clearly identified in the image analysis. This is due to the fact that in the upper sediment layer analysed in the sieve analysis sediment from the mobile armour layer is mixed with the fine sediment just underneath. This is the reason for the top sample resulting from sieve analysis to be finer than the top image. Except for this top layer, we find that the areal fraction content of fines derived by image analysis is somewhat larger than the corresponding volume fraction content based on sieve analysis. For the medium and coarse fractions, we find the opposite trend. As mentioned before, there are some intrinsic differences between the two methods. The same factors encountered by previous authors using image analysis (Sect. 3.2) are valid for our method and related to the facts that (1) the image analysis outcomes represent a more discrete elevation are compared to the ones of sieve analysis which represents a sediment layer; (2) the particle arrangement, such as the orientation and/or partial burial of the grains of the medium and coarse fractions, influences the relative areal presence of the particles on the bed surface, which affects the number of pixels of a certain colour in an image. It is likely that the medium and coarse grains are covered by several fine particles, so reducing their areal fraction content when compared to the volume fraction content resulting from sieve analysis. To account for these intrinsic differences a conversion model is needed.

Figure 15 shows an example of the result of size stratification measurement method proposed in this manuscript (Chavarrías et al., 2013, 2014). The graph shows the size stratification of the delta in which the colours represent the mean grain size in an area within the deposit equal to $0.34 \mathrm{~m} \times 0.06 \mathrm{~m}$ (lateral vs. streamwise distance) at every $1 \mathrm{~cm}$ over the vertical. Figure 15 demonstrates the strength of the developed size stratification measurement method as it results in an unprecedented spatial density of information on the grain-size distribution.

\subsection{Conversion from areal to volumetric fractions}

The proposed method has appeared to be a powerful tool to determine areal fraction contents within a deposit. We use a conversion model to convert these areal fractions resulting from image analysis into volumetric fractions; furthermore, for modelling purposes we sometimes need information on volume rather than areal fraction contents.

In general, the results of the areal sampling techniques (e.g. wax or clay sampling, image analysis) are not equivalent to the outcomes of sieving. The only procedure that leads to a grain-size distribution that is directly comparable to the results of sieve analysis is the grid by number technique (Kellerhals and Bray, 1971). The grid by number technique 

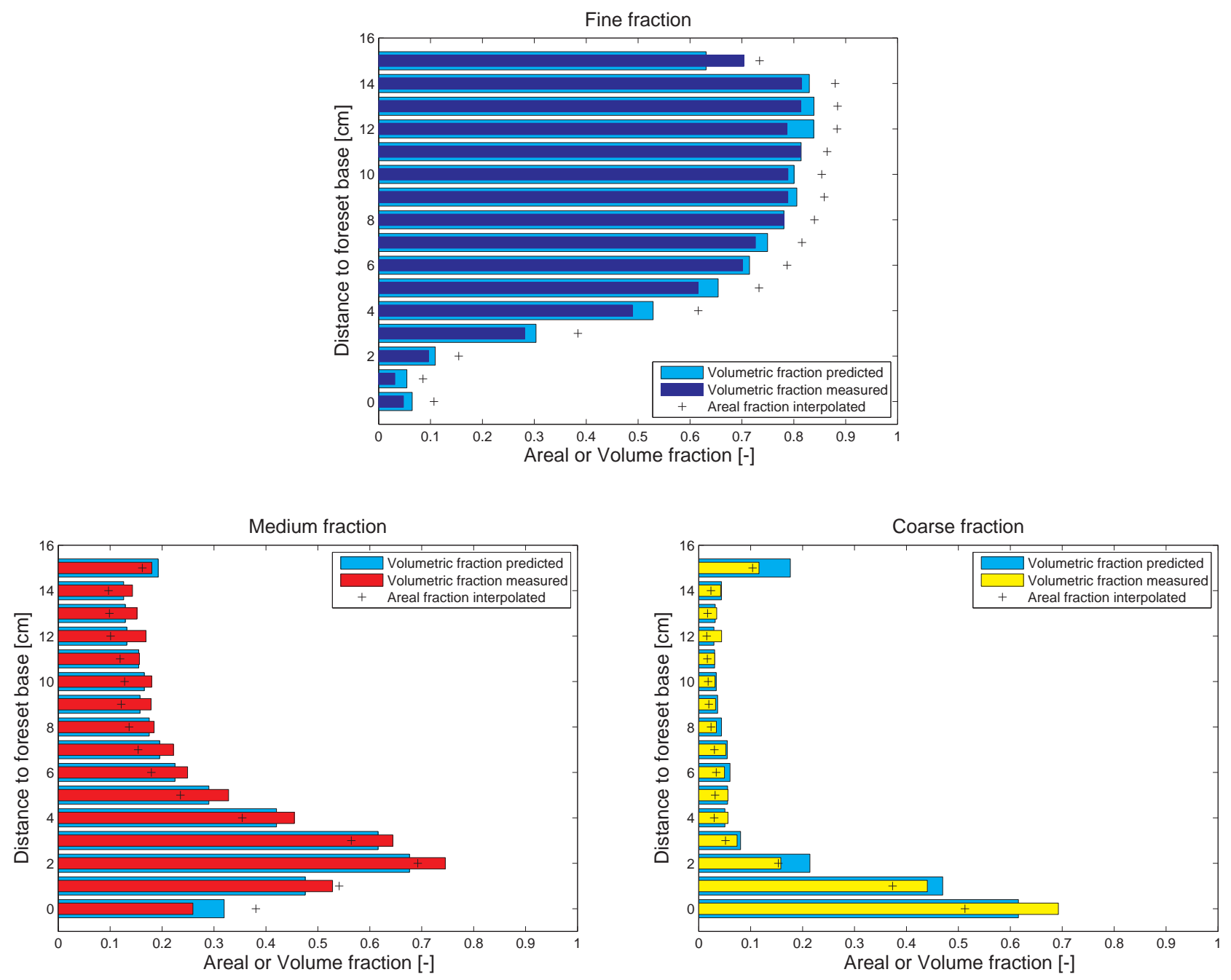

Figure 16. Vertical profiles of (1) the predicted volume fraction contents using Eq. (2), (2) the measured volume fraction contents measured through sieve analysis, (3) the areal fraction contents through image analysis for each grain-size fraction: (a) fine, (b) medium, and (c) coarse for Experiment 2, side sample. Note the areal fractions have been linearly interpolated. The crosses are now located at the elevation corresponding to the middle of the layer.

consists of collecting the particles below the nodes of a grid and counting the number of grains in the various size classes. The frequency of each size class is expressed as the percentage by number compared to the total number of particles. The method used to sample the particles in the grid by number is therefore equivalent to the resulting grain-size distribution (i.e. frequency by number). For the other areal sampling techniques (e.g. wax or clay sampling), the sampling methods are not equivalent to sieve analysis. Each sample has its own bias, which often not only depends on the method used for sampling and for the analysis, but also on the grain-size distribution of the deposit. Kellerhals and Bray (1971) were the first to provide a conversion model valid for a wide series of methods. They suggested an idealized cube to model the arrangement of mixed size particles without pores. Diplas and Sutherland (1988) modified the model to account for porosity. Fraccarollo and Marion (1995) present a conversion model that takes into account the irregular shape and random arrangement of the bed material. Therefore, unlike the cube model of Kellerhals and Bray (1971) and the modified version of Diplas and Sutherland (1988), the application of the model by Fraccarollo and Marion (1995) is not restricted to a fixed amount of grain-size fractions, certain particle sizes and shapes.

The conversion model used here is the one proposed by Parker (1991a, b). The model originates from the ones developed by Proffitt (1980) and Diplas and Sutherland (1988) and provides an estimate of the areal fraction content based on a given volume fraction content. The model by Parker (1991a, b) rewritten in the form as presented by Parker 


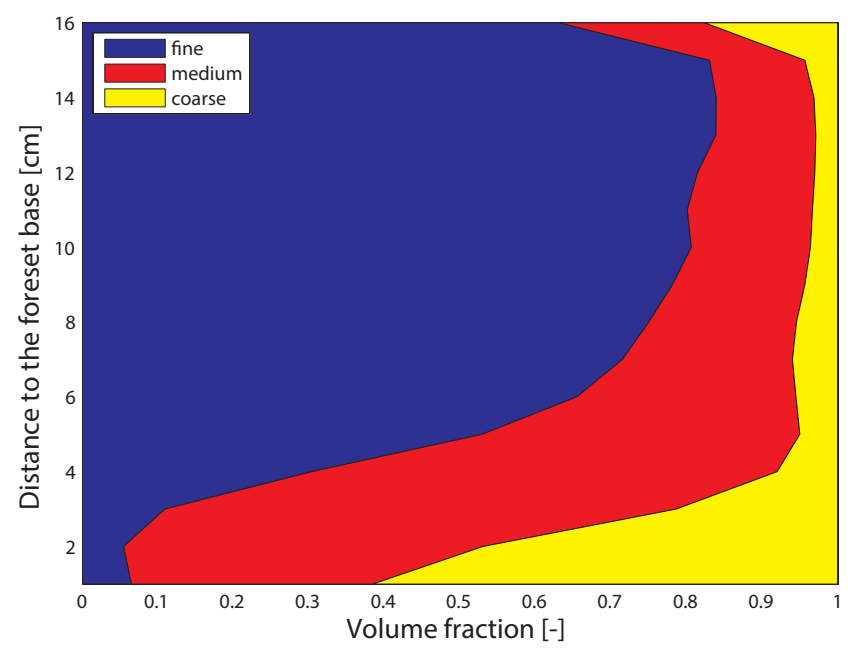

Figure 17. Vertical profile of the volume fraction contents for Experiment 2 (side sample) estimated through conversion of measured areal fraction contents to volume fraction contents using the Parker (1991a, b) conversion model.

and Cui (1998b) and Cui and Parker (2005) is

$$
F_{A i}=\frac{F_{V i} / \sqrt{D}_{i}}{\sum_{k=1}^{N}\left(F_{V k} / \sqrt{D}_{k}\right)},
$$

where $F_{V i}$ denotes the volume fraction content of size fraction $i$ in a sediment layer [-], $F_{A i}$ is the areal fraction content of size fraction $i$ of the same sediment layer [-], and $D_{i}$ is the grain size of size fraction $i[\mathrm{~m}]$. Like the subscript $i$, the subscript $k$ denotes the size fraction.

We now write Eq. (1) in the reverse form to determine the volume fraction content of each grain-size fraction, $F_{V i}$, from the areal fraction content resulting from image analysis, $F_{A i}$,

$F_{V i}=\frac{F_{A i} \sqrt{D}_{i}}{\sum_{k=1}^{N}\left(F_{A k} \sqrt{D}_{k}\right)}$.

Equation (2) was then applied to the data set of the Gilbert delta Experiments E1 and E2. The areal fraction contents resulting from image analysis were linearly interpolated between vertical elevations to provide data on the areal fractions at the same elevations as the values of the volumetric fraction contents. Figure 16 shows the results of the conversion model for the near-wall sample of Experiment E2. We now compare (1) the predicted volume fraction contents using Eq. (2), (2) the volume fraction contents measured through sieve analysis, and (3) the areal fraction contents determined through image analysis. Observing these three profiles, we notice that the conversion model reduces the overestimation of the fine fraction and the underestimation of the medium and coarse fractions. After converting the results of the image analysis into the corresponding volume fraction contents, we plot the variation of these volume fractions over the vertical (Fig. 17). We recognize a topset deposit over the elevation range between 15 and $16 \mathrm{~cm}$ from the foreset base. The topset is somewhat coarser than the sediment just below that belongs to the foreset deposit. The resulting foreset deposit is characterized by upward fining as a result of the avalanching process.

\section{Conclusions}

In this paper we present a new method to measure the entire size stratification of a sand-gravel deposit in a laboratory experiment. The method is based on a combination of particle colouring, removal of sediment layers, and image analysis. Colour segmentation of areal images of a bed surface covered by coloured particles is combined with a nondestructive and flexible method to remove sediment layers using a wet vacuum cleaner.

A clear advantage of the method is its high spatial resolution of the resulting data on the grain-size distribution, which is unequalled in other methods. Other methods generally provide information on the grain-size distribution in a limited number of locations.

We observed small inaccuracies in the sediment removal technique which are mainly attributable to imprecisions in the exact thickness of a sediment layer. Disturbances caused by driving a box corer into the bed, such as compressing sediment layers and/or displacing grains to lower elevations, are avoided here.

Using the proposed method a large amount of data is quickly collected, time consuming sieve analyses are avoided, and the data is quickly processed using an automatic and fast script. The image analysis processing is based on colour segmentation within the Lab colour space which results in colour detection of all pixels in an image. The accuracy in determining the areal fraction contents using image analysis is of the order of $1-2 \%$. A conversion model has been applied to convert areal fraction contents derived by image analysis into volume fraction contents.

The image analysis method was successfully used to determine the grain-size distribution of a submerged bed surface, while creating no disturbances to the bed surface. Measurement of the grain-size distribution of the bed surface during a running laboratory experiment, at flows with transported sediment, is currently being tested by the author.

Particle colouring by itself appeared to be of great help in the interpretation of grain-size selective processes based on observations of the flume experiments.

Overall, the size stratification measurement method, despite the sum of inaccuracies in particle colouring, sediment removal, and image analysis, results in a significantly smaller inaccuracy in measuring the size stratification of a deposit than a box corer. 
Acknowledgements. This research is funded by scholarship 10.015 of the Dr ir Cornelis Lely Stichting and Aspasia scholarship 015.007.051 of the Netherlands Organisation for Scientific Research (NWO). The authors especially thank the technicians of the Environmental Fluid Mechanics Laboratory of Delft University of Technology for their assistance during the experiments.

Edited by: F. Metivier

\section{References}

Adams, J.: Gravel size analysis from photographs, J. Hydraulics Division, ASCE, 105, 1247-1255, 1979.

Alexander, J., Bridge, J. S., Cheel, R. J., and Leclair, S. F.: Bedforms and associated sedimentary structures formed under supercritical water flows over aggrading sand beds, Sedimentology, 48, 133152,2001

Allen, J. R. L.: Sedimentation to the lee of small underwater sand waves: An experimental study, J. Geol., 73, 95-116, 1965.

Ashworth, P. J. and Ferguson, R. I.: Interrelationships of channel processes, changes and sediments in a proglacial braided river, Geografiska Annaler, Series A, Phys. Geogr., 68, 361-371, 1986.

Bagnold, R. A.: The physics of blown sand and desert dunes, Methuen, New York, 1941.

Blom, A.: Different approaches to handling vertical and streamwise sorting in modeling river morphodynamics, Water Resour. Res., 44, W03415, doi:10.1029/2006WR005474, 2008.

Blom, A., Ribberink, J. S., and de Vriend, H. J.: Vertical sorting in bed forms: Flume experiments with a natural and a trimodal sediment mixture, Water Resour. Res., 39, 1025, doi:10.1029/2001WR001088, 2003.

Carbonneau, P. E., Lane, S. N., and Bergeron, N. E.: Catchmentscale mapping of surface grain size in gravel bed rivers using airborne digital imagery, Water Resour. Res., 40, W07202, doi:10.1029/2003WR002759, 2004.

Carbonneau, P. E., Bergeron, N. E., and Lane, S. N.: Automated grain size measurements from airborne remote sensing for long profile measurements of fluvial grain sizes, Water Resour. Res., 41, W11426, doi:10.1029/2005WR003994, 2005.

Carling, P. A. and Reader, N. A.: A freeze sampling technique suitable for coarse river bed material, Sediment. Geol., 29, 223-239, 1981.

Chavarrías, V., Blom, A., Orrú, C., and Viparelli, E.: Laboratory experiment of a mixed-sediment Gilbert delta under varying base level, RCEM Symposium, 9-13 June, Santander, Spain, Book of abstracts, 114 pp., 2013.

Chavarrías, V., Orrú, C., Viparelli, E., Martín-Vide, J. P., and Blom, A.: Size stratification of a laboratory Gilbert delta due to base level changes, J. Geophys. Res., submitted, 2014.

Cui, Y. and Parker, G.: Numerical Model of Sediment Pulses and Sediment-Supply Disturbances in Mountain Rivers, J. Hydraul. Eng., 131, 646-656, 2005.

Diplas, P. and Sutherland, A.: Sampling Techniques for Gravel Sized Sediments, J. Hydraul. Eng., 114, 484-501, 1988.

Dugdale, S. J., Carbonneau, P. E., and Campbell, D.: Aerial photosieving of exposed gravel bars for the rapid calibration of airborne grain size maps, Earth Surf. Process. Landforms, 35, 627 639,2010
Edmonds, D. A., Shaw, J. B., and Mohrig, D.: Topset-dominated deltas: A new model for river delta stratigraphy, Geology, 39, 1175-1178, 2011.

Fraccarollo, L. and Marion, A.: Statistical Approach to BedMaterial Surface Sampling, J. Hydraul. Eng., 121, 540-545, 1995.

Gardner, J. T. and Ashmore, P.: Geometry and grain size characteristics of the basal surface of a braided river deposit, Geology, 39, 247-250, 2011

Gilbert, G. K.: The topographic features of lake shores, US Geol Surv., Annu. Rep., 5, 75-123, 1885.

Gilbert, G. K.: Lake Bonneville, US Geol. Surv. Monogr., 1, 438 pp., 1890.

Gomez, B.: Representative sampling of sandy fluvial gravels, Sediment. Geol., 34, 301-306, 1983.

Graham, D. J., Reid, I., and Rice, S. P.: Automated sizing of coarse grained sediments: Image-processing procedures, Math. Geol., 37, 1-28, 2005a

Graham, D. J., Rice, S. P., and Reid, I.: A transferable method for the automated grain sizing of river gravels, Water Resour. Res., 41, W07020, doi:10.1029/2004WR003868, 2005b.

Graham, D. J., Rollet, A.J., Piégay, H., and Rice, S. P.: Maximizing the accuracy of image-based surface sediment sampling techniques, Water Resour. Res., 46, W02508, doi:10.1029/2008WR006940, 2010.

Heays, K., Friedrich, H., and Melville, B. W.: Re-Evaluation of image analysis for sedimentary processes research. In: International Association of Hydraulic Engineering-Asia Pacific Division, Auckland, 21-24 February, 2010a.

Heays, K., Friedrich, H., and Melville, B. W.: Advance particle tracking for sediment movement on river beds: A Laboratory study, 17th Australasian Fluid Mechanics Conference Auckland, New Zealand 5-9 December, 2010b.

Hirano, M.: River bed degradation with armouring, Trans. Jpn. Soc. Civ. Eng., 3, 194-195, 1971

Ibekken, H. and Schleyer, R.: Photo-sieving: A method for grainsize analysis of coarse-grained, unconsolidated bedding surfaces, Earth Surface Proc. Landforms, 11, 59-77, 1986.

Jain, S.: Armor or Pavement, J. Hydraul. Eng., 116, 436-440, 1990

Jonasson, A. and Olausson, E.: New devices for sediment sampling, Mar. Geol., 4, 365-371, 1966.

Julien, P. Y. and Anthony, D. J.: Bed load motion and grain sorting in a meandering stream, J. Hydr. Res., 40, 125-133, 2002.

Kellerhals, R. and Bray, D. I.: Sampling Procedures for coarse fluvial sediments, J. Hydr: Div., ASCE, 97, 1165-1180, 1971

Kleinhans, M. G.: Grain-size sorting in grainflows at the lee side of deltas, Sedimentology, 52, 291-311, 2005.

Leopold, L. B. and Wolman, M. G.: River channel patterns: Braided, meandering, and straight: US Geological Survey Professional Paper 282-B, 39-85, 1957.

Lunt, I. A. and Bridge, J. S.: Evolution and deposits of a gravelly braid bar, Sagavanirktok River, Alaska, Sedimentology, 51, 415432, 2004.

MacQueen, J.: Some methods for classification and analysis of multivariate observations, Proceedings of the Fifth Berkeley Symposium on Mathematical Statistics and Probability, Statistics, University of California Press, Berkeley, California, 1, 281-297, 1967. 
Marion, A. and Fraccarollo, L.: Experimental investigation of mobile armouring development, Water Resour. Res., 33, 14471453, 1997.

McEwan, I. K., Sheen, T. M., Cunningham, G. T., and Allen, A. R.: Estimating the size composition of sediment surfaces through image analysis, Proc. Inst. Civ. Eng. Water Mar. Energy, 142, 189-195, 2000.

Pallara, O., Froio, F., Rinolfi, A., and Lo Presti, D.: Assessment of strength and deformation of coarse grained soils by means of penetration tests and laboratory tests on undisturbed samples, Solid Mechanics and Its Applications, 2007, Geotechnical Symposium in Rome, 146, 201-213, 2006.

Parker, G.: Selective sorting and abrasion of river gravel, I: theory, J. Hydraul. Eng., 117, 131-149, 1991a.

Parker, G.: Selective sorting and abrasion of river gravel, II: applications, J. Hydraul. Eng., 117, 150-171, 1991b.

Parker, G. and Andrews, E. D.: Sorting of bed load sediment by flow in meander bends, Water Resour. Res., 21, 1361-1373, 1985.

Parker, G. and Cui, Y.: The arrested gravel front: stable gravel-sand transitions in rivers. Part 2: General numerical solution, J. Hydr. Res., 36, 159-182, 1998b.

Parker, G. and Klingeman, P.: On why gravelbed streams are paved, Water Resour. Res., 18, 1409-1423, 1982.

Postma, G.: Sea-level-related architectural trends in coarse-grained delta complexes, Sediment. Geol., 98, 3-12, 1995.

Proffitt, G. T.: Selective transport and armoring of non-uniform alluvial sediments, Report 80/22, University of Canterbury, Christchurch, New Zealand, 1980.

Ribberink, J. S.: Mathematical modelling of one-dimensional morphological changes in rivers with non-uniform sediment, Ph.D. thesis, Delft University, Delft, Netherlands, 1987.

Rubin, D. M.: A simple autocorrelation algorithm for determining grain size from digital images of sediment, J. Sediment. Res., 74, $160-165,2004$
Sibanda, E., McEwan, I., and Marion, A.: Measuring the structure of mixed grain size sediment beds, J. Hydr. Engin., 126, 347353, 2000.

Sime, L. C. and Ferguson, R. I.: Information on grains sizes in gravel-bed rivers by automated image analysis, J. Sediment. Res. 73, 630-636, 2003.

Walkotten, W. J.: An improved technique for freeze sampling streambed sediments, USDA For. Serv. Res. Note, PNW-281, 11 pp., 1976.

Warrick, J. A., Rubin, D. M., Ruggiero, P., Harney, J. N., Draut, A. E., and Buscombe, D.: Cobble cam: grain-size measurements of sand to boulder from digital photographs and autocorrelation analyses, Earth Surf. Process. Landforms, 34, 1811-1821, 2009.

Wilcock, P. R. and Crowe, J. C.: Surface-based transport model for mixed-size sediment, J.Hydraul. Eng., 129, 120-128, 2003.

Wilcock P. R. and McArdell B. W.: Surface-based fractional transport rates: Mobilization thresholds and partial transport of a sand-gravel sediment, Water Resour. Res., 29, 1297-1312, 1993

Wilcock, P. R. and McArdell, B. W.: Partial transport of a sand/gravel mixture, Water Resour. Res., 33, 235-245, 1997.

Wu, F. C. and Yang, K. H.: Entrainment probabilities of mixed-size sediment incorporating near-bed coherent flow structures, J. Hydraul. Eng., 130, 1187-1197, 2004.

Zaman, T.: [Saturation] Saturate or Desaturate an image in Matlab (http://www.timzaman.com/?p=545), licensed under a Creative Commons Attribution-Non Commercial-Share Alike 3.0 Unported License, 2011.

Zanke, U.: Über den Einfluss von Kornmaterial, Strömungen und Wasserstanden auf die Korngrossen von Transportörpern in offenen Gerinnen, Tech. Rep. 44, Mitt. Franzius Inst., Hannover, Germany, 1976. 